\title{
On Surjective Bing Maps
}

by

\author{
Hisao KATO and Eiichi MATSUHASHI
}

Presented by Czestaw BESSAGA

Summary. In [7], M. Levin proved that the set of all Bing maps of a compact metric space to the unit interval is a dense $G_{\delta}$-subset of the space of all maps. In [6], J. Krasinkiewicz independently proved that the set of all Bing maps of a compact metric space to an $n$-dimensional manifold $(n \geq 1)$ is a dense $G_{\delta}$-subset of the space of maps. In [9], J. Song and E. D. Tymchatyn, solving some problems of J. Krasinkiewicz ([6]), proved that the set of all Bing maps of a compact metric space to a nondegenerate connected polyhedron is a dense $G_{\delta}$-subset of the space of maps. In this note, we investigate the existence of surjective Bing maps from continua to polyhedra.

1. Introduction. In this note, all spaces are separable and metrizable, and all maps are continuous. We denote the unit interval $[0,1]$ by $\mathbb{I}$. An arc is a space which is homeomorphic to $\mathbb{I}$. If $X$ is a compact metrizable space and $Y$ is a space, $C(X, Y)$ denotes the space of all continuous maps from $X$ to $Y$ endowed with the sup metric. A compact metrizable space is called a compactum, and a continuum means a connected compactum. A continuum is said to be indecomposable if it is not the union of two proper subcontinua. A compactum is called a Bing compactum (or hereditarily indecomposable) if each of its subcontinua is indecomposable. A map is called a Bing map if each of its fibers is a Bing compactum. In [7], M. Levin proved the following theorem.

Theorem 1 (M. Levin [7]). For each compactum $X$, the set of all Bing maps from $X$ to $\mathbb{I}$ is a dense $G_{\delta}$-subset in $C(X, \mathbb{I})$.

Independently, J. Krasinkiewicz proved the following theorem.

2000 Mathematics Subject Classification: Primary 54F45, 54F15; Secondary 54C05, $54 \mathrm{C} 35,54 \mathrm{C} 50$.

Key words and phrases: hereditarily indecomposable continuum, Bing compactum, Bing map, Menger manifold, 0-dimensional map. 
Theorem 2 (J. Krasinkiewicz [6]). Let $X$ be a compactum and let $Y$ be an $n$-dimensional manifold $(n \geq 1)$. Then the set of all Bing maps from $X$ to $Y$ is a dense $G_{\delta}$-subset in $C(X, Y)$.

In [9], J. Song and E. D. Tymchatyn solved some problems of J. Krasinkiewicz $([6])$. In particular, they proved the following:

Theorem 3 (J. Song and E. D. Tymchatyn [9]). The set of all Bing maps of a compact metric space to a nondegenerate connected polyhedron (or a 1-dimensional locally connected continuum) is a dense $G_{\delta}$-subset of the space of maps.

In this note, we investigate the existence of surjective Bing maps from continua to polyhedra and other spaces. Let $X$ and $Y$ be compacta. Let $C_{S}(X, Y)$ be the set of all surjective maps in $C(X, Y)$. Note that $C_{S}(X, Y)$ is closed in $C(X, Y)$. Also, let $B_{S}(X, Y)$ be the set of all Bing maps in $C_{S}(X, Y)$. A map $f: X \rightarrow Y$ is called an $\varepsilon$-map if $\operatorname{diam} f^{-1}(y)<\varepsilon$ for each $y \in Y$, and is 0 -dimensional if $\operatorname{dim} f^{-1}(y) \leq 0$ for each $y \in Y$.

Now, we prove the following theorem.

Theorem 4. Let $X$ be a nondegenerate continuum and let $\mathbf{P}$ be a finite $n$-dimensional connected polyhedron $(n \geq 1)$. Then $B_{S}(X, \mathbf{P})$ is a dense $G_{\delta}$-subset in $C_{S}(X, \mathbf{P})$.

To prove this theorem, we need the following proposition. The proof is obtained by modifying a proof of the Dugundji extension theorem.

Proposition 5. Let $X$ be an n-dimensional compactum, $A$ a closed subset of $X$, and $\sigma^{n}$ an n-dimensional simplex. If $g: X \rightarrow \sigma^{n}$ is a map such that $g \mid A: A \rightarrow \sigma^{n}$ is 0 -dimensional, then for any $\varepsilon>0$ there is a 0 -dimensional map $\widetilde{g}: X \rightarrow \sigma^{n}$ such that $\widetilde{g}$ is an extension of $g \mid A$ and $d(\widetilde{g}, g)<\varepsilon$.

Proof. Let $C\left(X, \sigma^{n} ; \varepsilon\right)$ be the set of all maps $f: X \rightarrow \sigma^{n}$ such that if $y \in \sigma^{n}$ and $C$ is a component of $f^{-1}(y)$, then $\operatorname{diam} C<\varepsilon$. Also, let $C\left(X, \sigma^{n} ; 0\right)$ be the set of all 0-dimensional maps $f: X \rightarrow \sigma^{n}$. Set

$$
C\left(X, \sigma^{n}: g \mid A\right)=\left\{f \mid f \in C\left(X, \sigma^{n}\right) \text { and } f|A=g| A\right\} .
$$

We will prove that $C\left(X, \sigma^{n}: g \mid A\right) \cap C\left(X, \sigma^{n} ; \varepsilon\right)$ is a dense open subset of $C\left(X, \sigma^{n}: g \mid A\right)$. Put $G=X-A$. Let $f \in C\left(X, \sigma^{n}: g \mid A\right)$. Then there is a canonical open covering $\mathcal{G}$ of $G$ in $X$ such that $\operatorname{mesh}(\mathcal{G})(<\varepsilon)$ is sufficiently small and $\operatorname{dim} N(\mathcal{G}) \leq n$ (see [3, (1.4), p. 70]), where $N(\mathcal{G})$ denotes the nerve of the covering $\mathcal{G}$. Consider the space $Z=A \cup N(\mathcal{G})$, where the neighborhoods of a point $z \in Z$ are defined as subsets $U_{z}$ of $Z$ such that:

(a) If $z \in A-\operatorname{cl}(G)$, then $U_{z} \cap A$ is a neighborhood of $z$ in $X$.

(b) If $z \in N(\mathcal{G})$, then $U_{z} \cap N(\mathcal{G})$ is a neighborhood of $z$ in $N(\mathcal{G})$.

(c) If $z \in A \cap \operatorname{cl}(G)$, then $U_{z} \cap A$ is a neighborhood of $z$ in $A$ and there exists a neighborhood $V$ of $z$ in $X$ such that every simplex 
$\sigma=\sigma\left(G_{0}, G_{1}, \ldots, G_{k}\right) \in N(\mathcal{G})$, where $G_{0} \cup G_{1} \cup \cdots \cup G_{k} \subset V$, is contained in $U_{z}$ (see $[3$, p. 81]).

Then there is a canonical map $p: X \rightarrow Z$ such that $p \mid A=1_{A}: A \rightarrow A \subset Z$ and $p \mid X-A: X-A \rightarrow N(\mathcal{G})$ is an $\varepsilon$-map. Since $\operatorname{dim} N(\mathcal{G}) \leq n$, we may assume that there is a map $\psi: Z \rightarrow \sigma^{n}$ such that $\psi|A=g| A, d(\psi p, f)<\varepsilon$ and for each simplex $\sigma \in N(\mathcal{G}), \psi \mid \sigma$ is injective (see [3, p. 81]). Put $f^{\prime}=\psi p$. Since $f|A=g| A$ is a 0 -dimensional map and $p \mid X-A$ is an $\varepsilon$-map, we can see that $f^{\prime} \in C\left(X, \sigma^{n}: g \mid A\right) \cap C\left(X, \sigma^{n} ; \varepsilon\right)$. Clearly, $C\left(X, \sigma^{n}: g \mid A\right) \cap C\left(X, \sigma^{n} ; \varepsilon\right)$ is an open subset of $C\left(X, \sigma^{n}: g \mid A\right)$. By the Baire theorem,

$$
C\left(X, \sigma^{n}: g \mid A\right) \cap C\left(X, \sigma^{n} ; 0\right)=\bigcap_{k=1}^{\infty}\left(C\left(X, \sigma^{n}: g \mid A\right) \cap C\left(X, \sigma^{n} ; 1 / k\right)\right)
$$

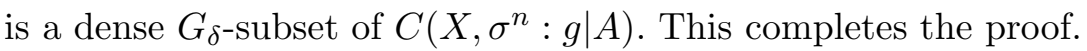

Proof of Theorem 4. Let $f \in C_{S}(X, \mathbf{P})$ and let $\varepsilon>0$. Choose a simplicial triangulation $\mathcal{K}$ of $\mathbf{P}$ such that $\operatorname{mesh}(\mathcal{K})$ is sufficiently small. By Theorem 3 , there is a Bing map $g: X \rightarrow \mathbf{P}$ such that $d(f, g)<\varepsilon / 2$ and for each principal simplex $T$ of $\mathcal{K}, g(X)$ intersects the interior $T-\partial T$ of $T$. Recall that a simplex $T \in \mathcal{K}$ is principal if it is a proper face of no simplex of $\mathcal{K}$. Let $T \in \mathcal{K}$ be a principal simplex of $\mathcal{K}$. Since $g(X)$ is a continuum, we can take a Cantor set $C$ in $g(X) \cap(T-\partial T)$. Put $E=T \cap g(X)$ and $F=(\partial T \cap g(X)) \cup C$. Take a surjective map $s: C \rightarrow T$. Define a map $h: F \rightarrow T$ by $h(x)=x$ for $x \in \partial T \cap g(X)$, and $h(x)=s(x)$ for $x \in C$. Note that $h$ is a 0 -dimensional map and $\operatorname{dim} E \leq \operatorname{dim} T$. By Proposition 5 , we obtain a 0 -dimensional surjective map $\widetilde{h}_{T}: E \rightarrow T$ with $\widetilde{h}_{T} \mid F=h$. By using the map $\widetilde{h}_{T}$ for each principal simplex $T$ of $\mathcal{K}$, we obtain a 0 -dimensional surjective map $\psi: g(X) \rightarrow \mathbf{P}$ such that $d\left(\psi, \mathrm{id}_{\mathbf{P}}\right)<\varepsilon / 2$. Put $g^{\prime}=\psi g: X \rightarrow \mathbf{P}$. Then $d\left(f, g^{\prime}\right)<\varepsilon$ and $g^{\prime}$ is the desired surjective Bing map. This completes the proof.

Theorem 6. Let $X$ be a continuum and let $Y$ be a Peano curve. Then $B_{S}(X, Y)$ is a dense $G_{\delta}$-subset in $C_{S}(X, Y)$.

Proof. Note that for each $\varepsilon>0$ there are a graph $G$ and surjective maps $p: Y \rightarrow G$ and $q: G \rightarrow Y$ such that $d\left(q \cdot p, \operatorname{id}_{Y}\right)<\varepsilon$ and $q$ is 0-dimensional. By using Theorem 4, we can easily get the assertion.

Corollary 7. Let $\mathbf{M}$ be a Menger manifold with $\operatorname{dim} \mathbf{M} \geq 1$. Then $B_{S}(X, \mathbf{M})$ is a dense $G_{\delta}$-subset in $C_{S}(X, \mathbf{M})$.

(See [1] for properties of Menger manifolds.)

To prove the above corollary, we need the following proposition.

Proposition 8. Let $X$ be an n-dimensional compactum and $A$ a closed subset of $X$. Let $\mathbf{M}$ be a Menger manifold with $\operatorname{dim} \mathbf{M}=n \geq 1$. If $g: X \rightarrow$ 
$\mathbf{M}$ is a map such that $g \mid A: A \rightarrow \mathbf{M}$ is 0-dimensional, then for any $\varepsilon>0$ there is a 0 -dimensional map $\widetilde{g}: X \rightarrow \mathbf{M}$ such that $\widetilde{g}$ is an extension of $g \mid A$ and $d(\widetilde{g}, g)<\varepsilon$.

Proof. The proof is similar to that of Proposition 5. By the $Z$-set embedding theorem for Menger manifolds (see [1]), in the proof of Proposition 5 we may assume that $\psi \mid \sigma: \sigma \rightarrow \mathbf{M}$ is a $Z$-embedding into $\mathbf{M}$ for each $\sigma \in N(\mathcal{G})$.

Proof of Corollary \%. The assertion follows from the proof of Theorem 6, Theorem 4 and Proposition 8.

Corollary 9. Let $X$ be any nondegenerate continuum. If $Y$ is either a compact nondegenerate connected polyhedron, a Peano curve, or a Menger manifold, then there is an upper semicontinuous decomposition $\mathcal{D}$ of $X$ such that each element of $\mathcal{D}$ is a Bing compactum and the quotient space $X / \mathcal{D}$ of $X$ with respect to $\mathcal{D}$ is homeomorphic to $Y$.

Proof. Since $Y$ is a Peano continuum, $C_{S}(X, Y)$ is nonempty. Hence so is $B_{S}(X, Y)$.

Also, we give an application of Theorem 4. We need the following wellknown theorem.

Theorem 10 (M. Brown [4]). Let $\left\{X_{i}, f_{i}\right\}$ be an inverse sequence such that $X_{i}$ is compact for each $i=1,2, \ldots$ Then there exist $\varepsilon_{1}>\varepsilon_{2}>\cdots>0$ such that if $g_{i}: X_{i+1} \rightarrow X_{i}$ are maps such that $d\left(f_{i}, g_{i}\right)<\varepsilon_{i}$ for each $i$, then $\lim _{\longleftarrow}\left\{X_{i}, g_{i}\right\}$ is homeomorphic to $\lim _{\longleftarrow}\left\{X_{i}, f_{i}\right\}$.

By Theorems 4 and 10, we obtain the following result.

COROllary 11. For each nondegenerate continuum $X$, there exists an inverse sequence $\left\{P_{i}, g_{i}\right\}$ such that $P_{i}$ is a nondegenerate compact connected polyhedron and $g_{i}: P_{i+1} \rightarrow P_{i}$ is a surjective Bing map for each $i=1,2, \ldots$, and $X=\lim _{\longleftarrow}\left\{P_{i}, g_{i}\right\}$.

Problem 12. Does the assertion of Corollary 9 hold if $Y$ is any nondegenerate Peano continuum?

\section{References}

[1] M. Bestvina, Characterizing k-dimensional universal Menger compacta, Mem. Amer. Math. Soc. 380 (1988).

[2] R. H. Bing, Higher-dimensional hereditarily indecomposable continua, Trans. Amer. Math. Soc. 71 (1951), 267-273.

[3] K. Borsuk, Theory of Retracts, PWN, Warszawa, 1967.

[4] M. Brown, Some applications of an approximation theorem for inverse limits, Proc. Amer. Math. Soc. 10 (1960), 478-483.

[5] R. Engelking, Theory of Dimensions, Finite and Infinite, Heldermann, 1995. 
[6] J. Krasinkiewicz, On mappings with hereditarily indecomposable fibers, Bull. Polish Acad. Sci. Math. 44 (1996), 147-156.

[7] M. Levin, Bing maps and finite-dimensional maps, Fund. Math. 151 (1996), 47-52.

[8] S. B. Nadler Jr., Continuum Theory. An Introduction, Dekker, 1992.

[9] J. Song and E. D. Tymchatyn, Free spaces, Fund. Math. 163 (2000), 229-239.

Hisao Kato and Eiichi Matsuhashi

Institute of Mathematics

University of Tsukuba

Ibaraki, 305-8571 Japan

E-mail: hisakato@sakura.cc.tsukuba.ac.jp

matsuhashieiichi@mail.goo.ne.jp

Received December 30, 2003;

received in final form February 4, 2004 